\title{
Publisher Correction: On the issue of transparency and reproducibility in nanomedicine
}

Hon S. Leong, Kimberly S. Butler, C. Jeffrey Brinker, May Azzawi, Steve Conlan, Christine Dufès, Andrew Owen, Steve Rannard, Chris Scott, Chunying Chen, Marina A. Dobrovolskaia, Serguei V. Kozlov, Adriele Prina-Mello, Ruth Schmid, Peter Wick, Fanny Caputo, Patrick Boisseau, Rachael M. Crist, Scott E. McNeil, Bengt Fadeel, Lang Tran, Steffen Foss Hansen, Nanna B. Hartmann, Lauge P. W. Clausen, Lars M. Skjolding, Anders Baun, Marlene Ågerstrand, Zhen Gu, Dimitrios A. Lamprou, Clare Hoskins, Leaf Huang, Wantong Song, Huiliang Cao, Xuanyong Liu, Klaus D. Jandt, Wen Jiang, Betty Y. S. Kim, Korin E. Wheeler, Andrew J. Chetwynd, Iseult Lynch, Sayed Moein Moghimi, André Nel, Tian Xia, Paul S. Weiss, Bruno Sarmento, José das Neves, Hélder A. Santos, Luis Santos, Samir Mitragotri, Steve Little, Dan Peer, Mansoor M. Amiji, Maria José Alonso, Alke Petri-Fink, Sandor Balog, Aaron Lee, Barbara Drasler, Barbara Rothen-Rutishauser, Stefan Wilhelm, Handan Acar, Roger G. Harrison, Chuanbin Mao, Priyabrata Mukherjee, Rajagopal Ramesh, Lacey R. McNally, Sara Busatto, Joy Wolfram, Paolo Bergese, Mauro Ferrari, Ronnie H. Fang, Liangfang Zhang, Jie Zheng, Chuanqi Peng, Bujie Du, Mengxiao Yu, Danielle M. Charron, Gang Zheng and Chiara Pastore

Correction to: Nature Nanotechnology https://doi.org/10.1038/s41565-019-0496-9, published online 3 July 2019.

In the version of this Correspondence originally published, Christine Dufès was incorrectly written as Christine Dufés. This has been corrected in the online versions of the Correspondence. 